\title{
Simulation Analysis and Study of Comprehensive Stress Change Regularity In the Sintering Process of LTCC Microwave Component
}

\author{
Benye Yin ${ }^{1, a}$, Zhaohua Wu ${ }^{1, b}$ \\ ${ }^{1}$ Electromechanical Engineering College, Guilin University of Electronic Technology, Guangxi, \\ Guilin, 541004, China \\ a752954365@qq.com, bemezdj@guet.edu.cn
}

Keywords: LTCC microwave component, sintering, technological parameter, comprehensive stress

\begin{abstract}
LTCC microwave component has small spacing between lines, the tiny deformation in sintering will affect the mechanical properties of components seriously. This paper established a 3D finite element simulation analysis model of the LTCC microwave component with micro-channels by using the software ABAQUS, due to the higher requirements of temperature control in the process of sintering, here simulated the sintering process by the choice of three process parameters like heating rate, holding time and cooling rate which had a greater influence on sintering shrinkage. About the LTCC component of microwave, the research had shown that in the phase of sintering densification, cooling rate is the most significant the influence on comprehensive stress, substrate contracts much more severely when there is a faster cooling rate, it causes local stress concentration, deformation, and even cracking; A faster heating rate will produce more liquid phase, pores can't discharge, affecting the densification of the component; reasonable extension of the holding time can promote the sintering process, but a too long holding time will aggravate the secondary recrystallization, then the densification will be inadequate along with the deformation. A set of reasonable sintering process parameters is obtained by simulation analysis: the heating rate is $6^{\circ} \mathrm{C}$ $/ \mathrm{min}$, the holding time $12 \mathrm{~min}$, the cooling rate is $9^{\circ} \mathrm{C} / \mathrm{min}$, which can avoid the stress concentration phenomenon effectively, and improve the mechanical performance of the components.
\end{abstract}

\section{Introduction}

With the miniaturization, integration and high frequency of electronic components, which requires the substrate of integrated circuit has the characteristics, such as high transmission speed, high density of wiring, etc. LTCC material has been widespread concerned and gets more and more widely used because of its better characteristics of high frequency, high speed transmission, low sintering temperature $\left(<1000^{\circ} \mathrm{C}\right)$ and good compatibility.

The control of sintering shrinkage rate is one of the key technologies of LTCC substrate manufacturing, for large area, multilayered LTCC substrate, the sintering shrinkage consistency has a great influence on the yield and the product performance. By controlling the influence factors in the process of sintering can control the sintering shrinkage rate effectively, The main factors include powder granularity, hot pressing laminated pressure and sintering curve etc. Sintering curve not only affect the performance of the substrate but also affect the sintering shrinkage rate. After the organic binder in the green ceramic tape is volatilized, heating rate, cooling rate and holding time will affect the sintering shrinkage rate directly. In this paper, a 3D model of LTCC microwave components is established by the finite element software ABAQUS, through simulating the process of sintering, studying the distribution of stress and the change rule in the process of sintering about the component, finally get the influence law of process parameters on comprehensive stress. 


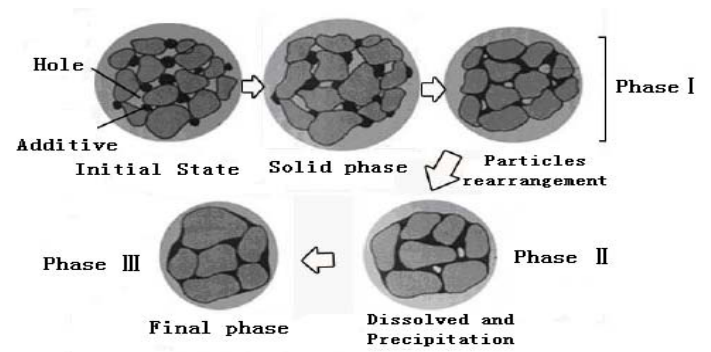

Fig.1 Sintering densification process

\section{Introduction of sintering process}

LTCC sintering process is heating fire the green ceramic tape in the sintering furnace according to a established sintering curve after it has been cut into pieces. The two phases mainly contained in sintering process are dumping and densification, time zone between $400^{\circ} \mathrm{C} \sim 500^{\circ} \mathrm{C}$ is called organic adhesive dumping area, and then to the high temperature $\left(500^{\circ} \mathrm{C} \sim 850^{\circ} \mathrm{C}\right)$ of sintering densification, the peak temperature will stay $8 \mathrm{~min} \sim 12 \mathrm{~min}$. This paper reflects the defects like warping and cracking due to shrinkage in sintering process by describing the change of comprehensive stress, the sintering densification process is showed in figure1.

Deformation of the sintered body is mainly caused by the viscous deformation, and viscous strain is far higher than the instantaneous elastic strain of sintered body, thus the effect of elastic strain in the sintered body can be ignored, the sintering of sintered body can directly equivalent for the sintering of isotropic viscous body, then a simple and effective formula for sintering obtained as follows:

$$
\dot{\varepsilon_{x}}=\dot{\varepsilon}_{f}+E_{p}^{-1}\left[\sigma_{x}-v_{p}\left(\sigma_{y}+\sigma_{z}\right)\right] ; \quad \dot{\varepsilon_{y}}=\dot{\varepsilon}_{f}+E_{p}^{-1}\left[\sigma_{y}-v_{p}\left(\sigma_{x}+\sigma_{z}\right)\right] ; \quad \dot{\varepsilon_{z}}=\dot{\varepsilon}_{f}+E_{p}^{-1}\left[\sigma_{z}-v_{p}\left(\sigma_{x}+\sigma_{y}\right)\right]
$$

In this formula, $\dot{\varepsilon}_{\text {is }}$ the strain rate, ${ }^{E_{p}}$ is the uniaxial viscosity of sintered body, ${ }_{p}$ is the viscous poisson coefficient of sintered body. The relationship between the stress and the densification rate of sintered body can directly get through the above formula.

\section{The establishment of simulation model}

Here the model is made by using the LTCC material-Ferro-A6M, the model has 25 layers and its size is $40 \times 40 \times 0.127 \mathrm{~mm}$. The main micro-channel size is $36 \times 2.2 \mathrm{~mm}$ and the secondary micro-channel size is $30.2 \times 1 \mathrm{~mm}$, micro-channels are stacked together after they were made respectively, and they were connected with each other. Sacrifice material adopts the polypropylene carbonate, embedded in micro-channel, and its size is $30.1 \times 0.9 \mathrm{~mm}$, It play a supporting role in the laminating process to prevent the deformation of micro-channel, it volatilizes in the dumping phase of sintering. The whole geometric model as shown in figure 2 , the sacrifice material model as shown in figure 3 , the related dimensions are shown in table 1.
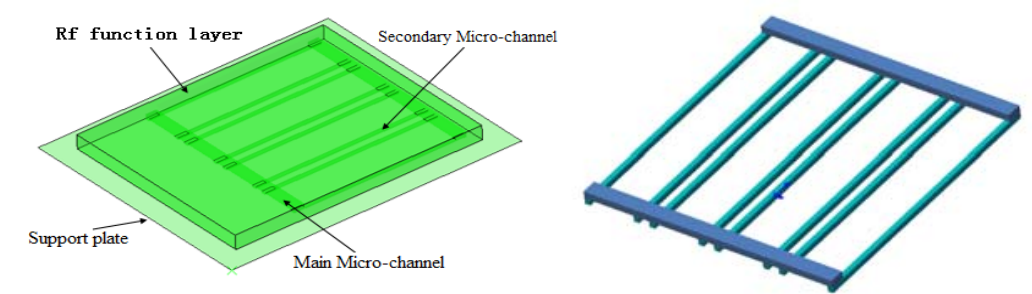

Fig. 2 The whole geometric model Fig. 3 The sacrifice material in micro-channel 
Table 1 The geometry size of the 3D mode

\begin{tabular}{ccccc}
\hline Size & $\begin{array}{c}\text { Green ceramic } \\
\text { tape }\end{array}$ & $\begin{array}{c}\text { Main } \\
\text { micro-channel }\end{array}$ & $\begin{array}{c}\text { secondary } \\
\text { micro-channel }\end{array}$ & $\begin{array}{c}\text { sacrifice } \\
\text { material }\end{array}$ \\
\hline Long $(\mathrm{mm})$ & 50 & 36 & 30.2 & 30.1 \\
Width $(\mathrm{mm})$ & 40 & 2.2 & 1 & 0.9 \\
Thickness $(\mathrm{mm})$ & 3.302 & 0.508 & 0.508 & 0.508 \\
\hline
\end{tabular}

The simulation analysis was used by general finite element analysis software ABAQUS and the entire model structure was divided by hexahedral C3D8T thermal coupling unit in this paper, mesh refinement appropriately in the micro-channel, the finite element mesh is shown in figure 4.

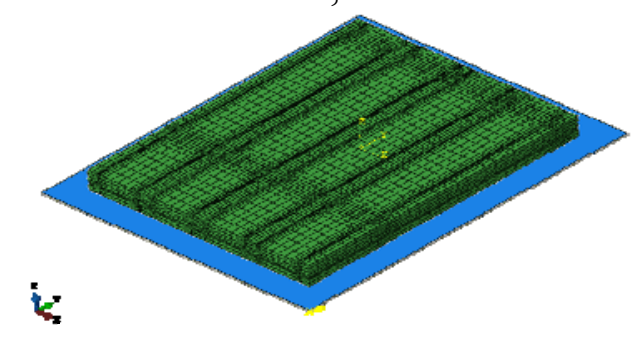

Fig.4 LTCC microwave modules finite element mesh model

LTCC sintering process is mainly a process of thermal effect, by heating the LTCC raw ceramics to reach densification and thus it has a certain strength and performance. The load boundary conditions include the initial reference temperature and heat convection. In ABAQUS, the initial temperature of each node is defined $25^{\circ} \mathrm{C}$, sintering heat transfer mainly considering heat convection and heat conduction. LTCC component was free without pressure, considering the contact between component and platform, meanwhile setting the acceleration of gravity is $9.8 \mathrm{~m} / \mathrm{s}^{2}$.

\section{The analysis of simulation results}

\subsection{The typical sintering curve and sintering process}

The material of the components mainly made up of alumina and glass composition. With the temperature increasing, glass composition showed great viscosity, at the same time many small gaps formed by the ceramic and glass particles before densification. Then with the temperatures rising, glass composition and alumina particles melting, gaps decreasing and the volume shrinking. The LTCC sintering curve and sintering densification curve as shown in figure 5, it shows the relationship between LTCC sintering temperature, shrinkage, density and the sintering time.
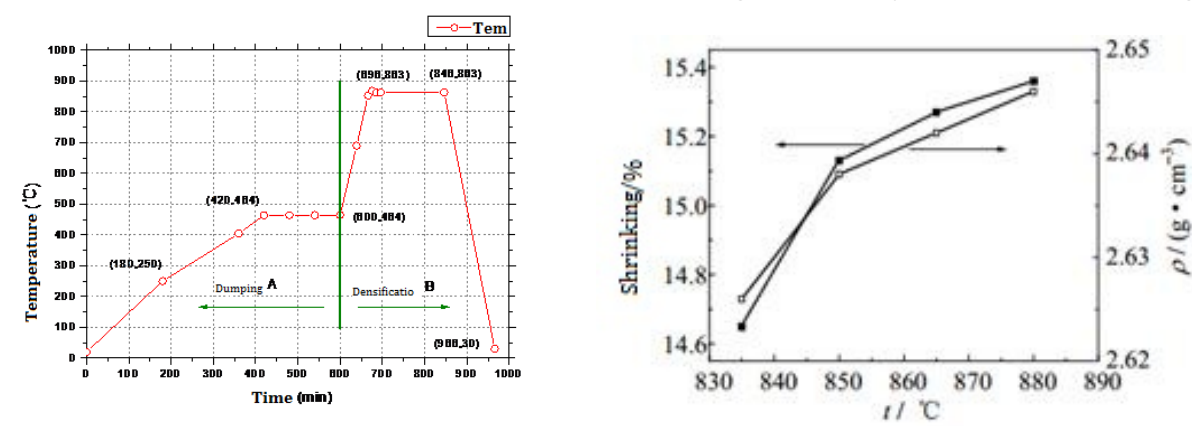

Fig.5 Sample sintering curve

The three main process parameters to be considered is heating rate, holding time and cooling rate. This paper analyses the stress distribution by the finite element simulation of typical process parameters, and comprehensive stress changing law of the component in the sintering process. The typical sintering process parameters were set: heating rate is $6^{\circ} \mathrm{C} / \mathrm{min}$, sintering holding time is $14^{\circ} \mathrm{C}$ $/ \mathrm{min}$, the cooling rate is $9^{\circ} \mathrm{C} / \mathrm{min}$. Total displacement nephogram and the stress distribution nephogram were extracted from the simulation result, which is shown in figure 6-7. 


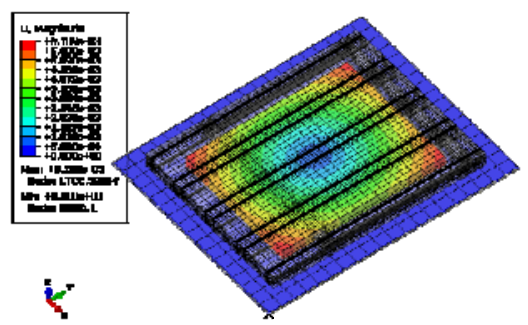

Fig.6 The total displacement nephogram of the 3D model after sintering

The picture shows that contraction is very obvious after sintering and four corners contract to the center. Since there are changes of displacement, there must be the change of stress. Below is the analysis of stress changing of LTCC microwave components. The nephogram shows in figure 7.

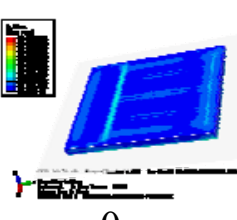

Os

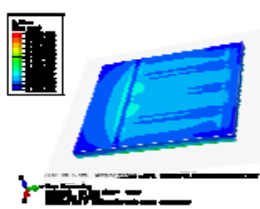

$360 \mathrm{~s}$

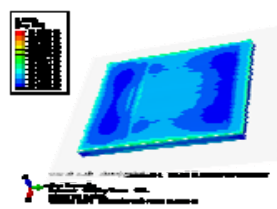

$1500 \mathrm{~s}$

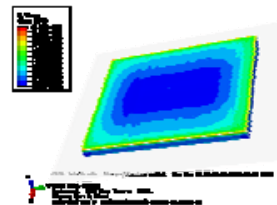

$1720 \mathrm{~s}$

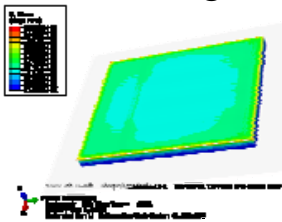

$2000 \mathrm{~s}$

Fig.7 The surface stress distribution and variation nephogram in sintering

These pictures show the surface stress of the components is increasing with the growth of sintering time. The contraction of ceramic substrate is the main reason for stress growth of external layer substrate. The stress of micro-channel will gradually become the minimum.

Stress concentration is obvious between micro-channels because of lamination process before sintering along with little deformation. During the sintering, there is stress relaxation and creep happening, residual stress caused by lamination is released. With densification increasing, the stress and deformation of the micro-channel recede. Stress will continue increase, but the stress in micro-channel will be the minimum in whole components gradually. The total stress distribution of LTCC Microwave components before sintering and sintering sample are showed in Fig 8.
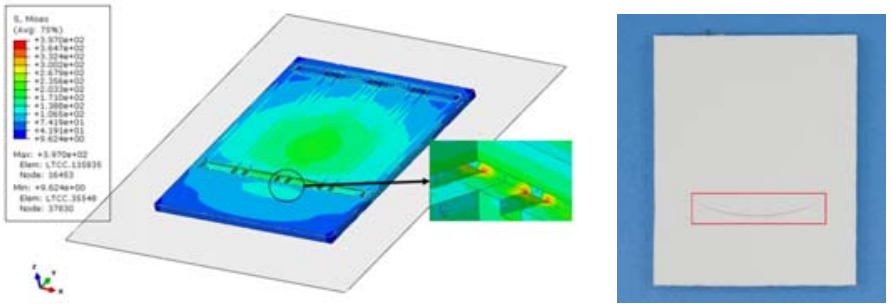

Fig.8. Micro-channel stress concentration phenomenon and sintered sample

\subsection{Effects of sintering process parameters on deformation of micro channels}

The effects of sintering process parameters based on typical process parameters, heating rate, holding time and cooling rate are separately changed to simulate. Heating rate contains 5, 6, 7 and $8^{\circ} \mathrm{C} / \mathrm{min}$; holding time contains $10,12,14$ and $16 \mathrm{~min}$; cooling rate contains $5,7,9$ and $11^{\circ} \mathrm{C} / \mathrm{min}$. the changing process of combined stress is showed in Fig9.
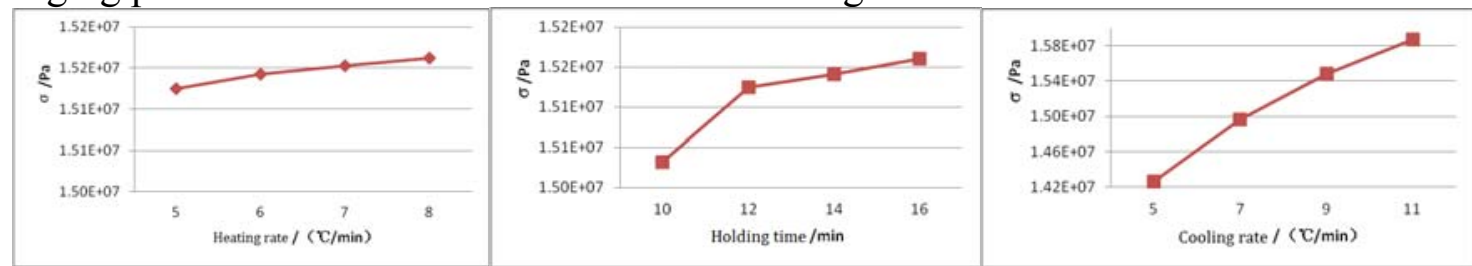

(a) Heating rate and stress (b) Holding time and stress (c) Cooling rate and stress

Fig.9 The relationship between comprehensive stress and various process parameters

It can be seen from above diagrams, the comprehensive stress showed a trend of continuous increasing in sintering, but the influence of different process parameters on comprehensive stress is different, the effect of cooling rate is the most significant, it presents almost a linear relationship between stress and cooling rate, the faster the cooling rate, the greater comprehensive stress changes; the holding time is relatively has a less influence, while the holding time reaches 14 min, the comprehensive stress reached $15.2 \mathrm{MPa}$, and tends to be stable; the influence of heating rate is 
minimum, as the heating rate increased, the comprehensive stress stays $15.2 \mathrm{MPa}$.

\section{Conclusion}

In this paper, the distribution of stress and comprehensive stress change rule about the specific LTCC microwave components in the process of sintering is studied by using the software ABAQUS simulation analysis. Results show that during the sintering densification process, volume shrinking, and the shrinkage rate of $\mathrm{x}$ axes, $\mathrm{y}$ axes and $\mathrm{z}$ axes reaches $17.2 \%$, the volume of the whole component decreases to $58.8 \%$; at the same time, the comprehensive stress increasing, the surface stress reaches $15.8 \mathrm{MPa}$, the stress concentration phenomenon is obvious; the stress of micro-channels is only $0.2 \mathrm{MPa}$, it will gradually become the minimum.

For all sintering process parameters, the influence of cooling rate on comprehensive stress is the most significant, by controlling the cooling rate under $10{ }^{\circ} \mathrm{C} / \mathrm{min}$ can effectively avoid the phenomenon of cracking due to thermal stress concentration in sintering. Heating rate has the least influence on comprehensive stress; setting the heating rate about $6^{\circ} \mathrm{C} / \mathrm{min}$, maintain a slow heating rate can improve the density of the component effectively; the comprehensive stress is increasing with the growth of sintering time; Keeping the sintering holding time of 12 min can avoid secondary recrystallization, thus can get sufficiently compact microwave component products.

\section{Acknowledgement}

This work is supported by National Defense Project of 973, a national defense preresearch project: 《The Study of $* * * * * * *$ Multiple Energy》. The corresponding author is Professor Zhao-hua Wu, Guilin University of Electronic Technology, email: emezdj@guet.edu.cn.

\section{Reference}

[1]He Jian-feng, LTCC Substrate Manufacture and Control [J]. Electronics Process Technology, 2005(02): p.75-81.

[2]Chen Lichun, Du Yongguo, Chen Xingyu, Zheng Xiaohui, Mechanical properties of LTCC green tape by tape-casting [J]. Electronic Components and Materials, 2009, 28(12): p.64-67.

[3]Wen Lin. Glass/ceramic low-temperature sinter material research [D].Hubei province: Material College, Huazhong University of Science and Technology, 2007.

[4] A. A. Shapiro, D. F. Elwell, P. Imamura, M. L.MeCartney, Structure-property relationships in low-temperature cofired ceramic, Proc. 1994 Int. Symp. On Microelectronics ISHM-94, Boston, 1994, p.306-311

[5]Bin Lin, Feng Liu, Xiaofeng Zhang, Liping Liu, Xueming Zhu, Simulation Technology in the Sintering Process of Ceramics[J]. Numerical Simulations-Applications, Examples and Theory, 2011: p. 401-414.

[6]Zhang, X. F; Lin, B \& Chen, S. H. (2008). Numerical Simulation of Temperature Distribution During Ceramic Sintering. Applied Mechanics and Materials Vols.10-12, p. 331-335. 